\title{
Analytic Computation Method of the Equivalent Thickness of Superposition Multi-Throttle-Slices of Twin-Tubes Shock Absorber
}

\author{
Changcheng Zhou, Yingzi Xu \\ School of Transport and Vehicle Engineering, Shandong University of Technology, Zibo, China \\ E-mail: greatwall@sdut.edu.cn
}

Received August 27, 2009; revised September 22, 2009; accepted October 4, 2009

\begin{abstract}
By elastic mechanics, the deformation of single throttle-slice for shock absorber was analyzed, the formula of its deformation was established. According to the relation of the deformation of multi-throttle-slices with the pressure on each slice, the analytic formula of equivalent thickness of multi-throttle-slices was established. Followed is a practical example for the computation of the equivalent thickness of multi-throttle-slices, compared the computed results with that simulated by ANSYS. The results show that the computation method of equivalent thickness of multi-throttle-slices is accurate enough.
\end{abstract}

Keywords: Twin-Tubes Shock Absorber, Multi-Throttle-Slices, Equivalent Thickness, Analytic Computation

\section{Introduction}

With the improvement of automobile technology, the velocity also improves, and there is a higher request to the smoothness and the security [1,2]. The characteristic of shock absorber influences driving smoothness and security of vehicles, nevertheless, it depends on the quality of the design and manufacture of shock absorber [3]. The telescopic twin-tubes shock absorber is widely used for vehicles. Nevertheless, it is still a puzzling problem for the throttle valves parameters design to calculate accurately the equivalent thickness of muti-throttle-slices. At present, both in domestic and abroad, in despite of many scholars analyzed the computation of equivalent thickness of multi-throttle-slices mostly by the finite element methods, they given out some qualitative conclusions only. This situation is un-accommodated to highly developed technology of automobile and affects the design quality of shock absorber. For the parameters design of throttle valves, there is not any analytic method yet, only with the experience of designer, testing and modification repeatedly $[4,5]$. It is said that the design for parameters of multi-throttle-slices, firstly a parameter value is guesstimated on experience, then testing and modification time and again; at lastly, the design value is fixed. While one parameter changing, other parameters would also change. Therefore, this method is inaccurate, the parameters of multi-throttle-slices could not been designed reliably. The traditional method of the equivalent-thickness of multi-th- rottle-slices is only a numerical simulation value [6,7], but it is unable to offer an analytic formula used to design the parameters of multi-throttle-slices.

In this paper, the analytic computation method of equivalent thickness of multi-throttle-slices was researched, the formula of equivalent thickness was established, and the results computed were tested with software ANSYS.

\section{Deformation of Single Throttle-Slice}

\subsection{Mechanics Model of Single Throttle-Slice}

Figure 1 is the mechanics model of elastic throttle slice. The boundary conditions of throttle slice are fixation restriction at the inner radius, and free restriction at the outer radius.

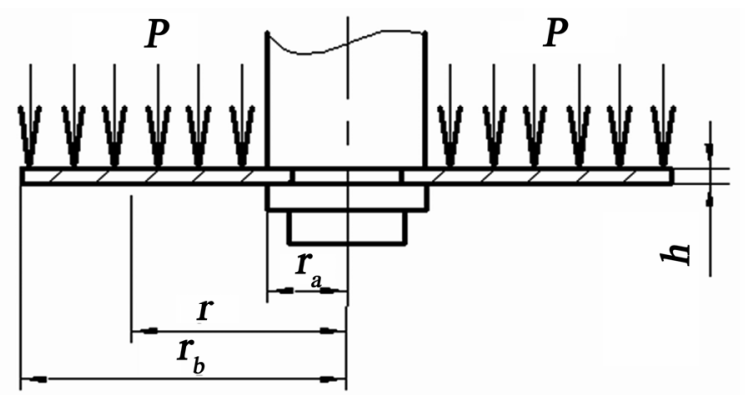

Figure 1. Mechanics model of elastic throttle-slice. 
where, $r_{a}$ is the inner radius, $r_{b}$ is the outer radius, $h$ is the thickness, $p$ is the pressure, and $f_{r}$ is the deformation at radius $r$.

\subsection{Math Model of Throttle-Slice Deformation}

Being symmetrical about the $z$-axis, the load and the structure, according to the basic principles in the elastic mechanics, the differential equation of elastic throttle slice deformation [8] is established as

$$
D\left(\frac{\mathrm{d}^{2}}{\mathrm{~d} r^{2}}+\frac{1}{r} \frac{\mathrm{d}}{\mathrm{d} r}\right)\left(\frac{\mathrm{d}^{2} f_{r}}{\mathrm{~d} r^{2}}+\frac{1}{r} \frac{\mathrm{d} f_{r}}{\mathrm{~d} r}\right)=p
$$

where, $D=E h^{3} /\left[12\left(1-\mu^{2}\right)\right] ; r$ is the any radius of throttle slice, $r \in\left[r_{a}, r_{b}\right] ; E$ is the elasticity coefficient of material of throttle slice; $\mu$ is Poisson rate; $h$ is the thickness of throttle slice. So, the solution of (1) is as

$$
f_{r}=\mathrm{C}_{1} \ln r+\mathrm{C}_{2} r^{2} \ln r+\mathrm{C}_{3} r^{2}+\mathrm{C}_{4}+\frac{3\left(1-\mu^{2}\right) r^{4} p}{16 E h^{3}}
$$

where, $\mathrm{C}_{1}, \mathrm{C}_{2}, \mathrm{C}_{3}, \mathrm{C}_{4}$ could be defined by the boundary conditions of throttle slice.

Therefore, analyzed the solution of the differential equation of slice deformation, the each items of it has the common factor of $p / h^{3}$ that can be bringing forward from (2), and then the deformation of throttle slice at any radius $r$ can be obtained.

$$
f_{r}=\left[\mathrm{K}_{\mathrm{C} 1} \ln r+\mathrm{K}_{\mathrm{C} 2} r^{2} \ln r+\mathrm{K}_{\mathrm{C} 3} r^{2}+\mathrm{K}_{\mathrm{C} 4}+\frac{3(1-\mu) r^{4}}{16 E}\right] \frac{p}{h^{3}}
$$

where, $\mathrm{K}_{\mathrm{C} 1}, \mathrm{~K}_{\mathrm{C} 2}, \mathrm{~K}_{\mathrm{C} 3}$ and $\mathrm{K}_{\mathrm{C} 4}$ are the residual parameters after the common factor $p / h^{3}$ is binging forward respectively from $\mathrm{C}_{1}, \mathrm{C}_{2}, \mathrm{C}_{3}$ and $\mathrm{C}_{4}$.

\subsection{Formula of Throttle-Slice Deformation}

Define $G_{r}$ as the deformation coefficient of throttle slice, it is as follow $[9,10]$

$$
\mathrm{K}_{\mathrm{C} 1} \ln r+\mathrm{K}_{\mathrm{C} 2} r^{2} \ln r+\mathrm{K}_{\mathrm{C} 3} r^{2}+\mathrm{K}_{\mathrm{C} 4}+\frac{3(1-\mu) r^{4}}{16 E}=G_{r}
$$

The $G_{r}$ is the inherent characteristic of throttle-slice deformation at radius $r$, denoting the deformation capability of throttle-slices.

So, the analytic formula of throttle-slice deformation at any radius was written briefly as

$$
f_{r}=G_{r} \frac{p}{h^{3}}
$$

For example, one throttle slice's $G_{r}$ is shown as in Figure 2.

\section{Equivalent Thickness of Multi-Throttle- Slices Superposition}

\subsection{Model of Multi-Slices Equivalent Thickness}

The sketch of the equivalent thickness of multi-throttle-

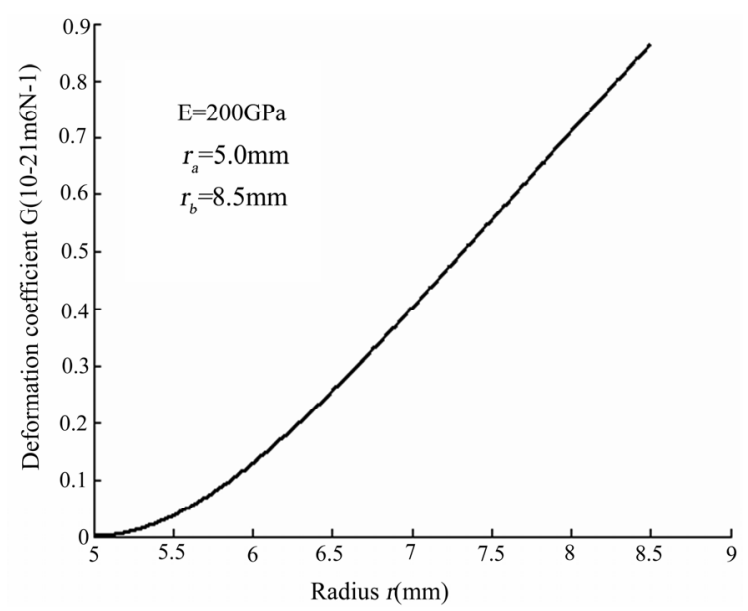

Figure 2. Curve of throttle slice deformation coefficient vs. radius.

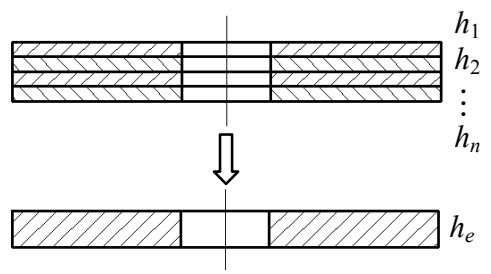

Figure 3. Sketch of multi-throttle-slices superposition.

slices superposition is shown in Figure 3.

\subsection{Formula of Multi-Slices Equivalent Thickness}

The model of throttle-slices superposition with unequal thickness could be taken as the paralleled springs that have the equal length, unequal elasticity coefficient.

The deformations of multi-slices are equal under same pressure. According to (5), it can be obtained such as

$$
G_{r} \frac{p_{1}}{h_{1}^{3}}=G_{r} \frac{p_{2}}{h_{2}^{3}}=\ldots . .=G_{r} \frac{p_{n}}{h_{n}^{3}}=G_{r} \frac{p}{h_{e}^{3}}
$$

The forces on multi-slices are unequal, but the summation of theirs is equal to the total force, i.e. $p_{1}+p_{2}+\ldots+p_{n}=p$.

Form (19), the equivalent thickness $h_{e}$ is written as

$$
h_{e}=\sqrt[3]{h_{1}^{3}+h_{2}^{3}+\ldots+h_{n}^{3}}
$$

If the each slice of multi-throttle-slices has multigroup thickness, i.e. $h_{1}, n_{2} ; h_{2}, n_{2} ; \ldots h_{n}, n_{n}$, so, (7) can be expressed

$$
h_{e}=\sqrt[3]{n_{1} h_{1}^{3}+n_{2} h_{2}^{3}+\ldots+n_{n} h_{n}^{3}}
$$

If the thickness of multi-throttle-slices is be equal each other, i.e. $h_{1}=h_{2} \ldots=h_{n}$, thus (7) can be written as

$$
h_{e}=h_{1} \sqrt[3]{n}
$$




\subsection{Equivalent Computation of Multi-Slices}

According to (7), the equivalent thickness of multi-slices can be computed, and the curve of equivalent thickness vs. slices number was shown as in Figure 4.

From (8) and Figure 4, it is known that the relations of the thickness of multi-slices with the equivalent thickness are as follows

1) The three power of the equivalent thickness is the sum of three power of each slice thickness.

2) The equivalent thickness of unequal thickness multi-slices is larger than the maximum thickness, i.e. $h_{\mathrm{e}}>\max \left[h_{i}\right]$.

3) The thickness of throttle-slice is standard, so, adapted to batch manufacture, and price depressed.

\section{Deformation of Multi-Throttle-Slices}

\subsection{Deformation}

For multi-throttle-slices $h_{1}, h_{2}, \ldots, h_{n}$, the equivalent

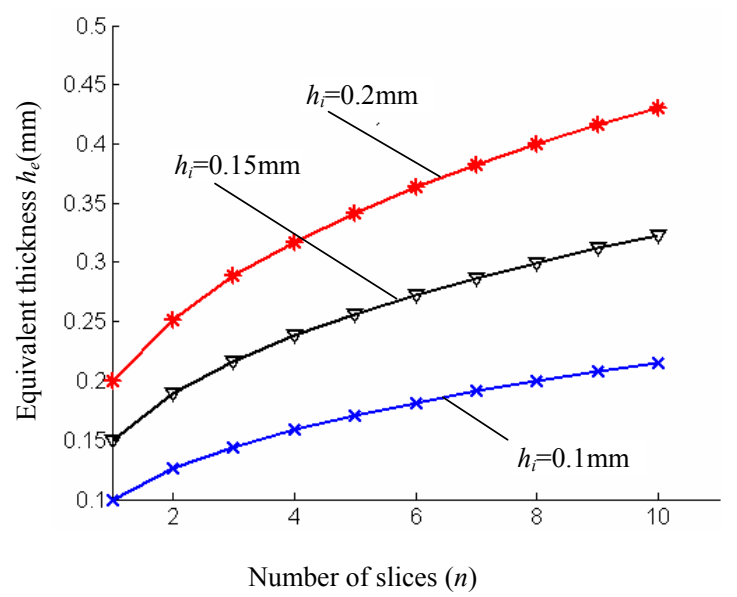

Figure 4. Curves of equivalent thickness at different slices number.

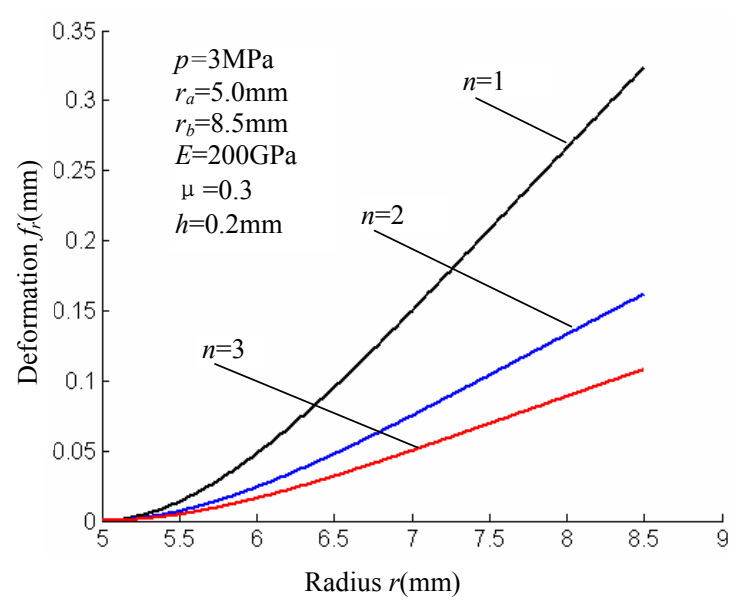

Figure 5. Deformation curve of multi-throttle slices superposition. thickness of them is $h_{e}$, the pressure loaded on mutithrottle-slices is $p$. The deformation of multi-throttle-slices could be regarded as the deformation of the single slice of thickness $h_{e}$.

According (5), the deformation of multi-throttle-slices can be expressed as

$$
f_{r}=G_{r} \frac{p}{h_{e}}
$$

Combining (10) with (7), so (10) is written as

$$
f_{r}=G_{r} \frac{p}{h_{1}^{3}+h_{2}^{3}+\ldots+h_{n}^{3}}
$$

From (11), it is known that the deformation of multislices is equal, as long as with different composing, but with the same equivalent thickness.

\subsection{Computation Example}

For example, if one single slice's thickness $h$ is $0.3 \mathrm{~mm}$, the multi-slices $h_{1}=0.2 \mathrm{~mm}, n_{1}=3$ and $h_{2}=0.1 \mathrm{~mm}, n_{2}=3$, the equivalent thickness $h_{e}$ is $0.3 \mathrm{~mm}$, the pressure $p=3 \mathrm{MPa}$. From (11), the deformation of multi-throttleslices is equal to that of single slice of thickness $h_{e}$.

For the multi-slices with different thickness, whereas the pressure is same, their deformation would be different. For example, the deformations computed by analytic formula of multi-slices with different equivalent thickness are as shown in Figure 5.

The computed deformation of throttle-slice is shown as in Table 1 .

\section{Simulation Certification}

The throttle slice model can be established with the finite element method by numeral software ANSYS, meshing with $0.1 \mathrm{~mm}$, loading and simulating.

The physical parameters of single throttle slice is as above, $h=0.3 \mathrm{~mm}$; and the multi-slices is $h_{1}=0.2 \mathrm{~mm}, n_{1}=3$ and $h_{2}=0.1 \mathrm{~mm}, n_{2}=3$, the equivalent $h_{e}$ is $0.3 \mathrm{~mm}$. Under pressure $3 \mathrm{MPa}$, the simulated results of deformation of single slice and multi-slices are as shown in Figure 6, and Figure 7, respectively.

From Figure 6, it is known that the maximum deformation simulated of single-slices is $0.126 \mathrm{~mm}$.

Compared with Table 1, the maximum deformation of single slice computed is very close to that simulated of multi-throttle-slices superposition, and their tolerance is only $0.03 \mathrm{~mm}$. It is shown that the computation method of single slice's deformation is accurate enough.

Table 1. Deformation of multi-throttle-slices at different radius $r$.

\begin{tabular}{cccccc}
\hline $\begin{array}{c}\text { Radius } \\
(\mathbf{m m})\end{array}$ & $\mathbf{5 . 0}$ & $\mathbf{6 . 0}$ & $\mathbf{7 . 0}$ & $\mathbf{8 . 0}$ & $\mathbf{8 . 5}$ \\
\hline $\begin{array}{c}\text { Coefficient } \\
\left(\mathbf{1 0}^{-22} \mathbf{m}^{\mathbf{6}} / \mathbf{N}\right) \\
\begin{array}{c}\text { Deformation } \\
(\mathbf{m m})\end{array}\end{array}$ & 0.000 & 1.350 & 4.207 & 7.467 & 0.909 \\
\hline
\end{tabular}




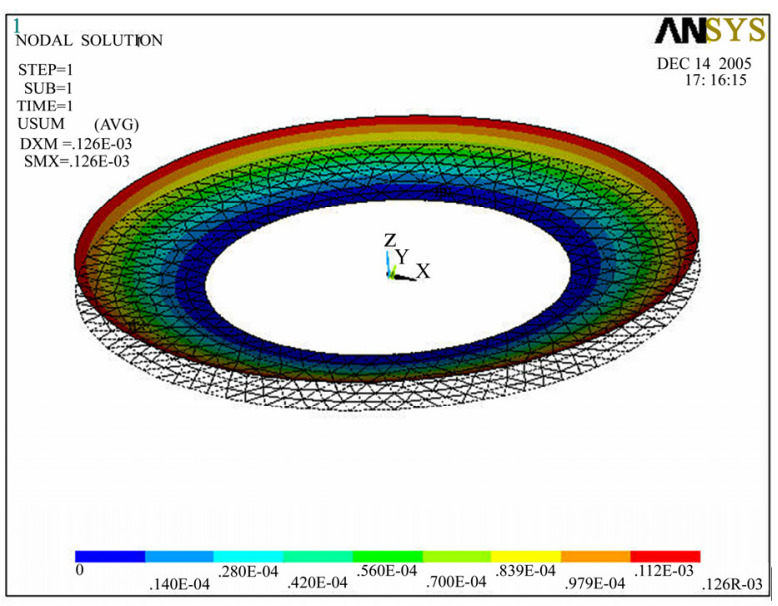

Figure 6. Deformation map simulated of single-throttle slices.

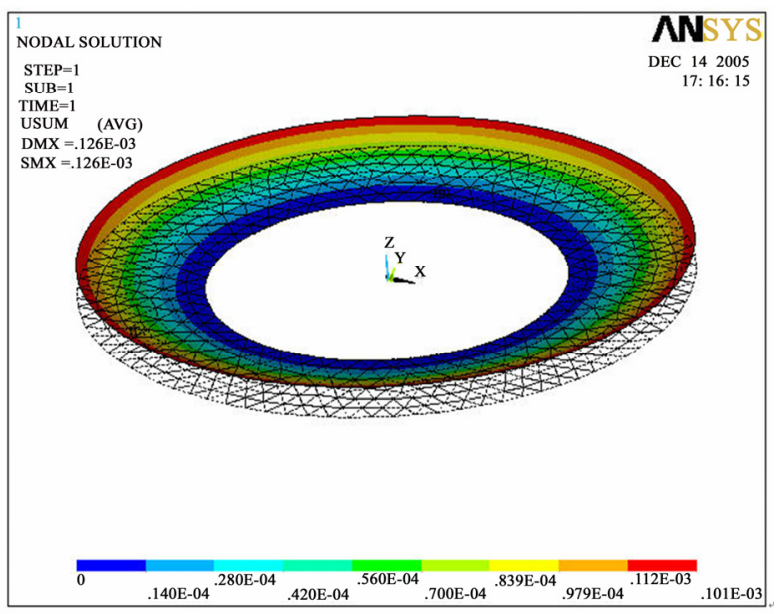

Figure 7. Deformation map simulated of multi-throttle-slices superposed.

From Figure 7, it is known that the maximum deformation simulated of multi-throttle-slices is $0.101 \mathrm{~mm}$ respectively.

Compared with Table 1, the maximum deformation computed of multi-throttle-slices superposition is close to that simulated, the tolerance is only $0.005 \mathrm{~mm}$. It is shown that the computation method of the equivalent thickness of multi-throttle slices superposition is accurate enough.

\section{Conclusions}

By the analytic computation and simulation certification to the equivalent thickness of multi-throttle-slices, it is known that the analytic formula of equivalent thickness of multi-throttle slices is accurate, simple and applied.
With it, the equivalent thickness of multi-throttle-slices with different thickness can be computed accurately.

The deformation computed of multi-slices is close to that simulated by ANSYS. It is shown that the computation method of the equivalent thickness of multi-throttleslices is accurate enough, can be used to design the parameters of throttle-slices of shock absorber.

\section{Acknowledgment}

The authors thank the Nature Science Foundation of Shandong (No. Y2007F72) for the funding support.

\section{References}

[1] D. F. Yu and Q. H. Chen, "Design study of smoothness-to-safety ratio in suspension shock absorber outer characteristic," Acta Armamentarll: The Volume of Tank, Armored Vehicle and Engine, Vol. 87, No. 3, pp. 11-17, 2002.

[2] C. C. Zhou, Z. Y. Zheng, and X. Y. Zhang, "Design method for throttle holes area of telescopic shock absorber for small electric vehicles," Journal of Asian Electric Vehicles, Vol. 7, No. 1, pp. 1191-1197, 2009.

[3] X. M. Fen and Z. M. Liu, "Development and current situation of automobile hydraulic telescopic damper technology," Journal of Wuhan University of Science and Technology, Vol. No. 8, pp. 340-343, 2003.

[4] T. Ashida, D. Tanaka and S. Minami, "A method to determine the velocity profiles from the power consumption of electric vehicles," Journal of Asian Electric Vehicles, Vol. 5, No. 2, pp. 1027-1032, 2007.

[5] S. Matsugaura, H. Nishimura, M. Omae, et al, "Development of a driver-monitoring vehicle based on an ultra small electric vehicle," Journal of Asian Electric Vehicles, Vol. 3, No. 2, pp. 758-762, 2005.

[6] S. M. Li, Z. H. Lü, "Technology development of cylindrical fluid drag shock absorber for motor vehicle," Automobile Technology, Vol. 32, No. 8, pp. 10-16, 2001.

[7] Y. Chen, H. E. Hui and J. F. Bai, "Analysis of hydraulic shock absorber spring valve chip deformation of charade car," Automobile Technology, Vol. 31, No. 1, pp. 19-21, 2000

[8] Z. L. Xu, "Elasticity mechanics," Beijing: Higher Education Press, 2001.

[9] C. C. Zhou, L. Gu, and L. Wang, "Bending deformation and coefficient of throttle-slice," Transactions of Beijing Institute of Technology, Vol. 26, No. 7, pp. 581-584, 2006.

[10] C. C. Zhou and L. Gu, "Superposition throttle slices opening size and characteristic test of telescopic damper," Chinese Journal of Mechanical Engineering, Vol. 43, No. 6, pp. 210-215, 2007. 